\title{
L'évaluation de la communication publique, entre norme gestionnaire et légitimités, des enjeux difficilement conciliables?
}

\section{Dominique Bessières}

\section{CpenEdition}

\section{Journals}

Édition électronique

URL : http://journals.openedition.org/communicationorganisation/1407

DOI : 10.4000/communicationorganisation. 1407

ISSN : $1775-3546$

Éditeur

Presses universitaires de Bordeaux

Édition imprimée

Date de publication : 1 décembre 2010

Pagination : 65-76

ISBN : 978-2-86781-743-4

ISSN : $1168-5549$

\section{Référence électronique}

Dominique Bessières, «L'évaluation de la communication publique, entre norme gestionnaire et

légitimités, des enjeux difficilement conciliables? », Communication et organisation [En ligne], 38 | 2010, mis en ligne le 01 décembre 2013, consulté le 10 décembre 2020. URL : http://

journals.openedition.org/communicationorganisation/1407; DOI : https://doi.org/10.4000/ communicationorganisation. 1407 


\title{
L'évaluation de la communication publique, entre norme gestionnaire et légitimités, des enjeux difficilement conciliables?
}

\author{
Dominique BESSIÈRES ${ }^{01}$
}

Le nouveau vocabulaire de l'action publique repose de façon visible sur des instruments de management public: pilotage par objectifs, gestion par résultats, gestion par projets ou par programmes, indicateurs de gestion et évaluation, recherche d'efficience... Ils illustrent les paradigmes sous-jacents véhiculés par l'instrument et la norme. D'un point de vue macro, le développement d'une gestion axée sur les résultats peut questionner l'essence même du service public, lequel est fondé sur des principes comme la neutralité, l'intérêt général...

À ce niveau, les réformes successives engagées depuis trois décennies dans les organisations publiques amènent à s'interroger sur le sens de ces innovations managériales introduites dans la sphère publique au regard de la thématique croissante de l'évaluation.

L'appréhension de ces enjeux heuristiques implique une analyse théorique mais également de terrain mobilisant des discours et documents d'acteurs (entretiens d'acteurs ${ }^{02}$, documentation...). Notre démarche compréhensive mobilise une grille pluridisciplinaire avec des éclairages théoriques issus des $\mathrm{SIC}$, de la sociologie des professions, de la science politique, des sciences de gestion.

01. Dominique Bessières est Maître de Conférences en Sciences de l'Information et de la Communication à I'Université de Reims Champagne-Ardennes. II est responsable de la spécialité de Master Communication publique et corporate de Sciences Po Lille. Mail : dominique.bessieres@iep.univ-lille2.fr

02. Issus d'une compilation de 68 entretiens semi-directifs (dont 11 femmes) diachroniques d'acteurs issus de différents terrains: responsables de communication, d'associations ou de salons professionnels, concepteurs de référentiels de compétences. Ils se sont déroulés entre 1993 et 2009, d'une durée de 40 minutes pour les plus brefs à 2 heures pour les plus longs, voire 3 heures dans quelques cas impliquant plusieurs entretiens. Ce corpus intègre plusieurs vagues et terrains, avec des questionnements communs sur leurs actions de communication menées, leurs contextes organisationnels spécifiques, leurs représentations de leur rôle l33 entretiens de responsables de la communication franciliens (1993-1997), 6 entretiens de responsables de communication publique champ'ardennais (2006-2008), 7 responsables de communication d'administration centrale (2006-2009), 15 directeurs de la communication de villes de province importantes (2006-2008), 7 entretiens de responsables d'associations professionnelles (1994, 2009). 
Fondamentalement, le transfert des modèles identifie les volontés normalisatrices (Bernard 1998, p. 58). L'idée de forme organisationnelle applicable à divers contextes en rend compte. Elle réfère à une permanence d'organisation en perpétuelle construction, à des logiques individuelles et collectives, à une médiation entre le local et le global (Bouillon, Bourdin, Loneux, 2008, p. 6). De sorte que des tendances générales, dans notre propos en faveur de l'évaluation du travail de la communication, vont trouver des modalités d'application au sein de certaines organisations, le cas échéant.

Les activités de communication institutionnelle représentent un cœur de métier des sciences de l'information et de la communication. Il n'en demeure pas moins que l'analyse de ses cadres d'évaluation dans le champ public doit intégrer la recherche de reconnaissance d'une professionnalité qui s’inscrit entre deux pôles: d'une culture métier et d'une culture des organisations publiques.

Derrière ces évolutions récentes, se pose en filigrane leur compatibilité avec l'idée de légitimité des acteurs. En effet, la légitimité politique a pu longtemps apparaître comme opposée aux visées d'évaluation de nature à remettre en cause potentiellement l'expression d'un pouvoir politique dans une zone de pouvoir organisationnel. Au départ et encore aujourd'hui, aucune obligation juridique n'impose la constitution de services de communication. À ce titre et dans leur périmètre, ils sont largement discrétionnaires. De plus, la légitimité professionnelle émergente des communicateurs publics (Bessières, 2009a) est directement concernée par une perspective d'évaluation.

La reconnaissance des fonctions récentes de communication met en jeu différentes sources de légitimités, alors même que leur rôle dans le fonctionnement des organisations publiques est plus fortement questionné aujourd'hui par la logique de performance recherchée par la $\mathrm{LOLF}^{03}$.

Aussi, dans quelles mesures les formes de management basées sur l'instrumentation de gestion ou le pilotage par les résultats sont-elles compatibles avec les démarches de communication orientées vers l'explicitation du sens? Pourquoi évaluer les actions de communications: s'agit-il d'un effet de mode ou d'une démarche de professionnalisation? Pourquoi une telle perspective est-elle encore peu développée?

\section{Le management signe de modernité professionnelle}

Au sein de l'État unitaire français, tout se passe comme si la logique d'adaptation de l'action intervient dans le développement des légitimités professionnelles.

03. Loi d'organisation relative aux lois de finance, 1er août 2001. 


\section{Un changement de représentation de l'action publique}

Le secteur public connaît une large diffusion d'une vision entrepreneuriale dès les 1980, devenue un nouveau critère de légitimité de l'action publique, dans l'optique de combattre des dysfonctionnements bureaucratiques. Son intérêt est d'introduire la préoccupation de performance finale davantage fondée sur l'action, rééquilibrant la classique conformité à la réglementation (Bartoli, 2009, p. 21-22). Elle manifeste une modification de la représentation de l'État. Ainsi, le management figure un langage adopté par les édiles au moment de la perte de légitimé économique du secteur public (Laufer, Burlaud, 1980, p. 36, p. 115) devant une vision de l'efficacité économique d'inspiration libérale. La légitimation des moyens ne peut plus se fonder sur le statut public. Ainsi, l'administration est amenée à rechercher un renouvellement de sa légitimité en permanence, en favorisant un consensus des usagers sur les fins et les résultats (Laufer, Paradeise, 1982, p. 42-43) au moyen de la communication.

La communication publique est, à maints égards, une traduction organisationnelle d'une préoccupation de management. Elle figure une mutation des relations avec les administrés de l'État qui recherche "une certaine qualité de contact. L'État est amené à utiliser de nouveaux procédés de gestion, y compris de gestion d'opinion "(Miège, 1989). Dans cette optique, la communication est mobilisée pour l'accueil du public, la présentation et la transmission d'informations, des campagnes visant à modifier des comportements (grandes causes d'intérêt général, sécurité routière...).

Aujourd'hui, la communication est intégrée par les acteurs dans la gestion. Le Vice-président du Conseil d'État (Sauvé, 2008) en souligne l'utilité pour peu qu'elle corresponde à l'accroissement du champ des services publics, aux sociétés contemporaines nécessitant une relation à la fois globale (idée de massification) et individuelle (idée de relations personnelles) avec le collectif et les personnes des usagers. Sous l'effet également des évolutions des contextes de fonctionnement, avec un approfondissement de la transparence administrative, la diffusion de méthodes de gouvernance négociatrices d'origine européenne liées à la communication, le développement de la consultation et de la participation (lois de 1995 et 2002).

Lintérêt organisationnel de la communication réside dans son intervention sur les représentations accompagnant des actions, des applications de règles, des procédures, la prise de décision publique. Elle met en forme des obligations d'information de certaines décisions (publicité des délibérations du conseil municipal), promeut des actions des services délivrés, ou fait connaitre l'institution par des campagnes ou actions d'intérêt général. L'image de l'action relève de la communication. Son rôle est à la fois modeste, parce que second par rapport aux actions de politiques publiques, mais important symboliquement parce qu'elle les accompagne pour les faire connaître et accepter. 


\section{Une interpénétration de l'État et des professions}

Certes, la thématique des normes implicites ou explicites d'appréciation des pratiques de travail est une antienne. Mais, le développement des modalités modernes d'organisation a renforcé l'impact des «investissements de formes" qui accompagnent aujourd'hui une certaine banalisation des pratiques de gestion des processus. Dès lors, il nous semble que la question de l'évaluation des pratiques professionnelles est particulièrement intéressante au niveau des communicateurs publics au croisement d'enjeux principiels et transversaux dans les organisations publiques. La communication publique en France est spécifiée (Bessières, 2009b) par le critère juridique du secteur public (son statut) et/ou sa nature non concurrentielle (son comportement).

En raison d'un mouvement « d'interpénétration de l'État et des professions » résultant de la nouvelle gestion publique (new public management), on note l'influence croissante de stratégies professionnelles dans l'action publique. L'analyse de la mise en œuvre des politiques publiques va de pair aujourd'hui avecl'importance du développement adaptatif de professionnalité au sein d'une administration de production (Le Bianic, Vion, 2008). Ce type de logique d'adaptation de l'action étatique intervient directement dans le renforcement de légitimités professionnelles en mobilisant des « experts» dans des cadres procéduraux en développement. Les statuts rigides sont ainsi amenés à être assouplis pour substituer aux solutions standardisées la contingence adaptée du terrain. La rationalité managériale s'avère plus prégnante, au point que les outils de gestion apparaissent aujourd'hui constitutifs de l'action publique. Ceci est patent au niveau de la communication publique et de son groupe professionnel.

\section{La norme d'évaluation difficilement assimilable}

La nouvelle gestion publique postule le développement de l'évaluation qui heurte l'organisation publique classique.

\section{Une nouvelle norme de gestion publique}

On mesure l'importance du développement en France de la nouvelle gestion publique qui oriente l'administration vers la performance (output) et non plus sur les ressources (input) (Finger M, Ruchat B 1997). Les professionnels de la communication publique en sont bien conscients, ils associent leurs pratiques aujourd'hui à une dimension de rigueur procédurale (organisation, prévision, gestion...) axée sur du management et de l'évaluation (Marchal, 2008, p. 347).

D'un point de vue global, la performance est à relier au développement de professionnalités et surtout à des diplômes dans la sociologie des professions (Dubar, Tripier 2005, p. 83-84). En effet, la reconnaissance universitaire permet d'acquérir des connaissances académiques (critère de performance) qui cessent d'être empiriques (apprentissage). En ce sens, les injonctions croissantes d'évaluation représentent des contraintes, mais également un 
support de reconnaissance de la professionnalisation de la communication publique. Dans un contexte de tension sur le niveau des dépenses publiques, ce qui se mesure peut être mieux défendu dans un contexte organisationnel: "L'évaluation en tant que mesure de l'utilité des actions de communication est sans doute un moyen de continuer à dégager des moyens financiers à l'avenir $»^{04}$.

Dans ce contexte, la légitimité même de la communication dans le fonctionnement des organisations publiques est concernée par la logique de performance véhiculée par la $\mathrm{LOLF}^{05}$. Le but principal réside dans la recherche d'un meilleur pilotage des dépenses et d'une responsabilité accrue des gestionnaires. Les budgets sont votés suivant des programmes fondés sur des objectifs mesurables assortis d'indicateurs de résultat. Toutefois, le concept de performance, figure moderne de l'évaluation dans la sphère publique, est difficile à cerner avec précision. Étymologiquement, il renvoie à l'idée d'accomplir une action, sans évoquer la nature, le niveau ou la mesure du résultat (Bartoli 2009, p. 105-106, p. 22). Dans une perspective instrumentale, évaluer les actions de communication, c'est se donner les moyens de rendre compte de l'utilisation des ressources, de mesurer les progrès mais aussi d'améliorer l'efficacité des actions. À ce titre, la nécessité plus forte de rendre compte n'est pas qu'un effet de mode, avec l'influence forte de la LOLF au nom des citoyens destinataires des politiques publiques. Les indicateurs de performance sont des mesures pour l'objectif de réduction des coûts. La performance et ses outils d'évaluation ont un coût sociopolitique de nature à expliquer les conditions difficiles de leur traduction sur le terrain de la communication.

\section{Une évaluation culturellement ardue}

La communication est souvent perçue comme non directement productive, rendant son évaluation difficile. Les méthodes d'évaluation se heurtent à des pratiques publiques hostiles à considérer des « clients " précisément ciblés qui privilégient un ou des grands publics relevant de découpages de compétences administratives générales (Bessières, 2000). Au total, la perspective de l'évaluation des actions de communication apparaît tardive. Si bien que sa difficulté se traduit dans la grande diversité des méthodes utilisées le cas échéant, sans qu'il soit besoin de les recenser de façon exhaustive. Des communicateurs publics ${ }^{06}$ indiquent la nécessité d'intégrer cette dimension en amont de l'action, les pré-tests et les post-tests à l'instar des communications commerciales ou des campagnes nationales publicitaires, des sondages. Or, le risque est présent de confondre l'évaluation de la communication aux effets diffus, avec l'évaluation des politiques publiques aux effets tangibles. À ce

04. Entretien de l'auteur dans une grande ville de province en 2009 ,

05. Loi d'organisation de la loi de finance

06. Résultant de plusieurs entretiens de l'auteur 
titre, les méthodes instrumentales d'évaluation peuvent sembler inadéquates. On se fie ainsi à des démarches empiriques et descriptives (ex. bouche-àoreille, comptabilisation des reprises presse ou des clics sur internet...), avec des recueils d'informations plus ou moins sommaires sans avoir toujours défini de valeur de référence. Aussi, les évaluations systématiques des actions de communication sont encore peu présentes.

Enfin, l'évaluation peut-elle viser seulement une action de communication ou est-elle intrinsèquement liée à l'appréciation de la politique publique décidée par des édiles qu'elle promeut? Elle repose toujours sur une décision des dirigeants politiques, faute d'obligation juridique pour la mise en place des services de communication (Bessières 1998, p. 64-65, p. 193, p. 225-226, 2009a). Au sein des collectivités locales, ils sont majoritairement rattachés aux plus hauts sommets hiérarchiques, directement auprès de l'exécutif ou via son cabinet. Une de nos enquêtes sur les responsables de communication franciliens ${ }^{07}$ montre que la majorité des services de communication sont une composante du cabinet, ou bien ont des liens forts avec lui dans l'organisation des décisions de communication. La forte présence de contractuels, aux positions statutaires moins assurées que des fonctionnaires titularisés, pour des postes hiérarchiques de communication l'atteste. On peut trouver davantage de fonctionnaires dans des postes moins stratégiques.

Cet état de fait traduit la prise en compte d'enjeux sociétaux ${ }^{08}$ intégrés de façon croissante depuis le tournant des années 1980 par les édiles dans les organisations administratives. En raison de leur jeunesse, les services fonctionnels transversaux ${ }^{09}$ de communication n'ont pas une grande légitimité historique. Pour autant, ils sont censés améliorer l'efficience globale et constituent un champ de compétences précis. Luttant contre la compartimentation administrative, ils veillent à organiser une certaine cohérence symbolique et imagière entre les composantes opérationnelles. Leur stratégie de reconnaissance va jusqu'à des positionnements de « clientfournisseur » auprès de l'exécutif et de l'organisation administrative (BessièresGrima 1999, p. 33-34), à l'exemple du Service commun d'Information et de la Communication du Ministère de l'Économie et des Finances (SIRCOM) depuis deux ans.

Pour autant, fondamentalement, cette activité de communication organisationnelle publique a toujours une signification politique. Elle est placée sous le contrôle d'un homme politique (ministre, élu) ou d'un dirigeant tirant sa légitimité d'une nomination politique. Elle sera jugée sur son impact dans l'opinion, sur l'image de l'institution, voire sur celle du dirigeant politique placé à sa tête (Laufer, Burlaud, 1980). Cette communication est

07. Entretiens de responsables de la communication franciliens (1993-1997)

08. Thèmes de la société de communication et de l'information caractérisant la société post-industrielle par sa généralisation dans tous les champs sociaux

09. Comme d'autres services fonctionnels à forte technicité: les formateurs, les services informatiques... 
de facto conditionnée par la notion de mandat, à ce titre elle est politique. Mais la question même de son évaluation dans ce contexte pose un problème ontologique en opposition à la notion de légitimité politique du pouvoir exécutif représentatif décideur des actions et comptable de leur communication. La logique de centre de coût discrétionnaire joue à l'encontre de l'évaluation en la matière. Cependant, le coût financier de telles opérations est souvent avancé pour ne pas les entreprendre ${ }^{10}$, comme également la difficulté de déterminer des objectifs de communication. Pour certains praticiens «au sein des collectivités territoriales les freins à 1 évaluation de la communication publique sont davantage culturels que techniques» .

Au niveau des collectivités locales, nous avons pu mesurer que la question de l'efficacité ou de l'inefficacité de la communication ne la remet pas en cause dans la mesure où elle est orientée vers le territoire local, sorte de marché captif (Bessières, 1998). Des universitaires y voient la dimension de la communication la plus difficile à connaître, tant au niveau de sa réception que de son acceptation (Tudesq, 1992), d'autant que des communications locales équivalentes peuvent se solder par des résultats contrastés (Faure, 1994), voire qu'il n'y a pas de savoirs stabilisés en la matière (Duran, Monnier, 1992, p. 236).

Au demeurant, au sein d'un de nos corpus des grandes collectivités locales franciliennes dans les années $1990^{11}$, nous n'avons rencontré qu'un seul cas d'évaluation des communications systématiquement menée en raison de l'opinion personnelle du directeur de la communication (Bessières 1998, p. 431-449).

Les seules mesures des différentes communications s'avèrent ainsi difficiles à isoler des actions et politiques publiques qu'elles promeuvent, de tous les autres supports avec lesquels elles fonctionnent en synergie, auprès de grands publics. On note une grande difficulté à cerner les effets imputables à la seule communication, dans une logique gestionnaire mettant en scène les actions publiques.

Différents positionnements en matière d'évaluation sont possibles sur un "continuum », avec d'un côté la conformité aux lois, règlements et choix politiques (modèle bureaucratique administratif classique) et de l'autre le pilotage par les résultats quantitatifs et financiers, laissant de nombreuses marges d'appréciation dont les acteurs peuvent ou pas se saisir (modèle gestionnaire actuel).

\section{L'évaluation entre expérimentation et basculement}

Le contexte public n'est pas homogène en terme de pratique d'évaluation, ce qui rend compte de la diversité du secteur public. Les différentes

10. Issus de plusieurs entretiens de l'auteur, notamment dans les collectivités locales franciliennes (19931997), champardennaises (2006-2008), de villes importantes $(2006,2009)$

11. Sur 33 entretiens de responsables de la communication franciliens (1993-1997) 
organisations qui le composent réagissent différemment. On va de la tradition d'une opposition, à des expérimentations avancées dont il faut mesurer les conditions.

\section{Une évaluation globale recherchée peu présente}

Dans une perspective de légitimation professionnelle, certains communicateurs publics s'avèrent parfaitement conscients de l'importance de présenter la communication comme une activité de management qui à ce titre implique une recherche d'efficacité, voire de performance et d'évaluation. Dans un équilibre à trouver entre les indicateurs de résultats et de coûts, certains pointent 4 grands critères d'évaluation d'une opération de communication "l'agrément, la notoriété, l'attribution, la compréhension " (Marchal, 2008, p. 349).

D'un point de vue instrumental, la démarche d'évaluation d'une action de communication n'est pas aisée et repose sur certains principes méthodologiques que l'on peut résumer à grands traits. L'évaluation se prévoit avant l'action et nécessite des objectifs de communication mesurables. Mais, les choix des indicateurs couvrent-ils l'ensemble des objectifs? Sont-ils faciles à établir, fiables et clairs? Leur coût est-il proportionné au regard de leur intérêt? Quel peut-être le temps de la mesure (avant, pendant, après) et selon quelle méthode quantitative ou qualitative? On voit bien au travers de ce simple questionnement la difficulté réelle des opérations d'évaluation.

Les premiers indicateurs de performance associés à la communication, issus d'une application de la LOLF, apparaissent impropres à retracer l'ensemble des activités communicationnelles parce qu'ils ne concernent qu'une information globale pour tout le service de communication le cas échéant. On note l'empirie et une grande diversité de critères choisis. Ils sont pour beaucoup auto-définis par des acteurs de la communication publique. De plus, comme ils doivent représenter les "productions » sous forme chiffrée et en même temps permettre de mesurer des marges de progression, on remarque qu'ils sont pour l'instant et en conséquence de type macro (ex. taux de notoriété pour la Direction générale pour l'armement depuis 2006, taux de connexion internet pour le Service d'information du gouvernement en 2006). En ce sens, ces indicateurs sont modestes.

Bien plus, dans la plupart des ministères on n'a pas choisi de faire entrer la communication explicitement dans le champ d'évaluation des indicateurs de performance. En conséquence, la communication publique est généralement regroupée au sein des budgets "affaires générales ", fonctions supports non stratégiques au sens de la LOLF au même titre que le personnel, l'informatique, l'immobilier... 


\section{Des exemples d'évaluation sous contrainte de qualité et de coût}

Les premiers exemples d'évaluation systématiques ont un caractère pionnier et singulier. C'est le cas de la Direction générale pour l'armement qui a entrepris de retracer une forme d'évaluation par reporting à la suite d'une politique de qualité avec certification ISO 9001. Son application initiée en 2002 fonctionne avec une évaluation de satisfaction (questionnaire) pour les communications événementielles, en interne en termes de respect des délais et des coûts.

En 2008, un nouveau directeur de la communication issu du privé a été nommé Chef du service de la communication commun aux ministères de l'Économie et du Budget. Sa mission a porté en particulier sur la mise en place d'une logique très directement inspirée du secteur privé où il a développé toute sa carrière jusqu'alors. Il a structuré une batterie d'indicateurs regroupant une petite centaine de sous-mesures d'évaluation consolidées au sein d'indicateurs (voir tableau ci-dessous).

\begin{tabular}{|c|c|}
\hline $\begin{array}{l}\text { Objectif: Améliorer l'information des publics } \\
\text { et faciliter les relations de l'usager avec } \\
\text { l'administration }\end{array}$ & $\begin{array}{l}\text { Objectif: Améliorer la qualité de service } \\
\text { auprès des commanditaires }\end{array}$ \\
\hline $\begin{array}{l}\text { Indicateurs: } \\
\text { - Taux de notoriété moyen des projets ou } \\
\text { actions ministériels auprès du grand public } \\
\text { - Taux de connaissance déclaré par les } \\
\text { agents des évolutions ministérielles }\end{array}$ & $\begin{array}{l}\text { Indicateurs: } \\
\text { - Taux de satisfaction global } \\
\text { - Aide à la définition des besoins } \\
\text { - Adéquation du produit aux attentes } \\
\text { - Respect des délais annoncés } \\
\text { - Réactivité et prise en compte des } \\
\text { imprévus } \\
\text { - Suivi et dialogue }\end{array}$ \\
\hline
\end{tabular}

L'objectif de contrôle des coûts est clairement affiché par son responsable ${ }^{12}$, lequel a d'ailleurs procédé à des redéploiements d'effectifs se traduisant par une très forte décrue du personnel attaché à ce service (de l'ordre de $50 \%$ ). On comprend alors que l'évaluation est ainsi conçue comme un moyen, pour l'ancien Ministre du Budget Woerth, au service de ses objectifs généraux de politique publique organisationnelle de diminution du nombre des fonctionnaires. La rationalisation technique et professionnelle portée par l'évaluation est également instrumentalisée dans une optique de réduction des dépenses. Le Ministre de l'Économie Lagarde semble plus sensible à une mobilisation satisfaisante des supports de communication. Tout se passe comme si les objectifs et indicateurs ainsi schématisés traduisent les logiques organisationnelles d'un service de communication d'un Ministère regroupant l'action de plusieurs ministres et leurs préoccupations stratégiques. Plus globalement, on est en présence d'un modèle totalement importé d'une logique privée de contrôle des coûts. Une telle systématicité de l'évaluation dépasse de beaucoup les objectifs portés par la LOLF, en termes d'étendue des dispositifs

12. Au cours d'une présentation du SIRCOM pour l'association Communication publique, 17 septembre 2010 
d'évaluation, tant par leur ampleur que par leur impact organisationnel, au profit d'une rationalisation forte autour d'expertises professionnelles voulues comme une agence de communication interne.

Ces exemples pionniers allant plus loin que les indicateurs de performance sont plus avancés que dans la plupart des institutions publiques, dans la mesure où ils correspondent à des objectifs managériaux globaux qui pèsent sur l'ensemble de ces organisations.

\section{Conclusion}

Globalement, la question du sens des évaluations des actions de communication se pose, notamment au regard de deux risques que nous avons décrits: un excès de contrôle procédurier que la nouvelle gestion publique traduite dans la LOLF voulait combattre, ou à l'inverse une évaluation forfaitaire peu précise, voire inexistante ancrée dans la culture des organisations publiques.

Nous sommes bien en présence de volontés normalisatrices qui s'expriment dans le transfert de modèles entre différents champs organisationnels, notamment du privé en direction du public. Pour autant, ces formes organisationnelles se diffusent dans la société en s'adaptant à différents champs tout en permettant une adaptation permanente et des innovations. Les organisations publiques dans leurs actions de communication sont ainsi aux prises avec des logiques globales et locales, c'est-à-dire collectives et individuelles. La légitimité professionnelle mais aussi politique des acteurs apparaît en filigrane.

Plus globalement, dans la continuité de ces évolutions, la question de l'articulation entre l'évaluation et la communication se pose également au travers des « rapports d'activité annuels ». Ceux-ci concernaient les entreprises vis-à-vis de leurs actionnaires et partenaires extérieurs. Récemment une directive du Premier ministre invite les ministères à établir un tel document vis-à-vis des parlementaires et de la presse, initiant ainsi un nouveau champ de communication publique: la communication financière.

\section{BIBLIOGRAPHIE}

BARTOLI A., Le management dans les organisations publiques, $3^{e}$ édition, Paris : Dunod, 2009.

BERNARD F. «Communication organisationnelle et usages des sciences humaines et sociales. Fragments de réflexion », in Communication d'entreprises et d'organisations, Le Moënne C. (Dir.), Rennes : PUR, 1998, pp. 49-78.

BESSIÈRES D., «La quête de professionnalisation des communicateurs publics entre difficulté et stratégie », Formation Emploi, n 108 octobre, décembre 2009, pp. 39-52. 
BESSIÈRES D., " La définition de la communication publique : des enjeux disciplinaires aux changements de paradigmes organisationnels ", Communication Eซ Organisation, $\mathrm{n}^{\circ}$ 35, Bordeaux : PUB, décembre 2009, p. 15-28.

BESSIERES D., «Un retour du local instrumentalisé », Paris : Quaderni, n 42, 2000, p. 5-16.

BESSIÈRES D., GRIMA F., «Quelles légitimités pour les services fonctionnels de la communication et de la formation dans les collectivités locales », Paris : Politiques et Management Public, volume 17, n 4/1999, p. 1-16.

BESSIERES D., L'institutionnalisation de la communication locale : le cas des échelons décentralisés départementaux, régionaux, parisiens franciliens. Thèse de science politique, Université Paris I Panthéon Sorbonne, 1998, 531 p.

BOUILLON J.-C., BOURDIN S, LONEUX C, «Approches communicationnelles des organisations : interroger l'organisation par la communication », Toulouse : Sciences de la société, no 74, PUM, mai 2008, p. 3-9.

DUBAR C., TRIPIER P., Sociologie des professions, Paris : Armand Colin, 2005.

DURAN P., MONNIER E., « Le développement de l'évaluation en France, nécessités techniques et exigences politiques ", Paris : RFSP, vol. 42, n 2, avril 1992, p. 235-262.

FAURE A., " Les élus locaux à l'épreuve de la décentralisation - de nouveaux chantiers pour la médiation politique locale », Paris : RFSP vol. 44, n³ 3, juin 1994 p. 462-479.

LAUFER R., BURLAUD A., Management public Gestion et légitimité. Paris : Dalloz, Coll. Gestion Systèmes et Stratégies, 1980, 353 p.

LE BIANIC T., VION A. (dir.), Action publique et légitimités professionnelles - Paris : LGDJ, col. Droit et société, avril 2008, 347 p.

FINGER M, RUCHAT B, « Le New Public Management : État, administration et politique ", pp. 33-56, in Pour une nouvelle approche du management public (réflexions autour de Michel Crozier), FINGER M., RUCHAT B. (dir), Paris : Ed. Seli Arslam, 1997, $252 \mathrm{p}$.

MARCHAL H, « Management et efficience », in LEMAIRE M. et ZÉMOR P. (dir.), La communication publique en pratiques, Paris : La documentation Française, 2008, p. 347-350.

MIEGE B., La société conquise par la communication, Grenoble : PUG, 1989, 226 p.

SAUVE J.-M., "Une exigence démocratique et un impératif de bonne gestion " in LEMAIRE M. et ZÉMOR P. (dir), La communication publique en pratiques, Paris : La documentation Française, 2008, p. 419-423.

TUDESQA.-J., "Conclusion », in " La communication dans l'espace régional et local », MABILEAU A., TUDESQA.-J. (dir.), Les cahiers du CERVEL, n 3, 1992, pp. 75-81. 
Résumé : L'évaluation de la communication publique est intéressante dans la mesure où elle articule plusieurs échelles, macro et micro, globale et organisationnelle, managériale et professionnelle. Dans quelle mesure cette nouvelle injonction à l'évaluation correspondelle à une visée normalisatrice et quel sens peut-on lui donner? Il s'agit d'un signe d'une modernisation de l'action publique qui heurte la culture du public à certains égards. Sa place s'inscrit dans un continuum allant d'une quasi-absence à des exemples d'une forte emprise.

Mots clés: Communication publique, indicateurs, légitimité, professionnalisation, nouvelle gestion publique

Abstract: The evaluation of public communication is interesting insofar as it articulates several scales, macro and micro, global and organizational, managerial and Professional. To what extent this new injunction in the evaluation correspond to a set and normalizing, what sense can we give? This is a sign of modernization of public action which offends the public culture in some extent. His place is a continuum ranging from a near absence to examples of a strong hold.

Key words : Public communication, indicators, legitimacy, profesionalization, new public management 